\title{
Analysis of hybrid dielectric-plasmonic slot waveguide structures with 3D Fourier Modal Methods
}

\section{J. Čtyroký}

\section{P. Kwiecien}

\section{Richter}

Recently, plasmonic waveguides have been intensively studied as promising basic building blocks for the construction of extremely compact photonic devices with subwavelength characteristic dimensions. A number of different types of plasmonic waveguide structures have been recently proposed, theoretically analyzed, and their properties experimentally verified. The fundamental trade-off in the design of plasmonic waveguides for potential application in information technologies lies in the contradiction between their mode field confinement and propagation loss: the higher confinement, the higher loss, and vice versa. Various definitions of figures of merit of plasmonic waveguides have been also introduced for the characterization of their properties with a single quantity. In this contribution, we theoretically analyze one specific type of a plasmonic waveguide - the hybrid dielectric-loaded plasmonic waveguide, or - as we call it in this paper - the hybrid dielectric-plasmonic slot waveguide, which exhibits very strong field confinement combined with acceptable losses allowing their application in some integrated plasmonic devices. In contrast to the structures analyzed previously, our structure makes use of a single low-index dielectric only. We first define the effective area of this waveguide type, and using waveguide parameters close to the optimum we analyze several waveguide devices as directional couplers, multimode interference couplers (MMI), and the Mach-Zehnder interferometer based on the MMI couplers. For the full-vector $3 \mathrm{D}$ analysis of these structures, we use modelling tools developed in-house on the basis of the Fourier Modal Method (FMM). Our results thus serve to a dual purpose: they confirm that (i) these structures represent promising building blocks of plasmonic devices, and (ii) our FMM codes are capable of efficient 3D vector modelling of plasmonic waveguide devices.

[DOl: http://dx.doi.org/10.2971/jeos.2013.13024]

Keywords: Plasmonic waveguides, hybrid dielectric-plasmonic waveguide, subwavelength confinement, Fourier modal method

\section{INTRODUCTION}

Recently, among a vast number of other plasmonic waveguides, a novel plasmonic waveguiding structure - a hybrid dielectric-loaded plasmonic waveguide (HDLPW) - has been proposed, its waveguide properties were numerically analyzed, and some passive and even also active plasmonic waveguide devices based on HDLPW were investigated both theoretically and experimentally [1]-[12]. This waveguide combines very strong mode field confinement with acceptably long propagation distances. In fact, we have recently independently arrived to a very similar structure in the course of testing the capabilities of modelling plasmonic structures with our full-vector 3D modelling tools developed on the basis of the Fourier Modal Method (FMM) [13]-[16]. Our thoughts were inspired by our previous work on silicon nanowire slot waveguides [17] that enable strong mode confinement in nonplasmonic structures, and were further directed to plasmonics by the idea of a PIROW waveguide [18]. The resulting waveguide combines the confinement of a surface plasmon with that of the electric field discontinuity at the dielectric interface; for this reason we suggest to call this waveguide - perhaps more aptly - a "hybrid dielectric-plasmonic slot waveguide" (HDPSW).
Although a number of different definitions of "figures of merit" have already been proposed [19] for characterization of plasmonic waveguides that combine both the mode field confinement and its propagation length, in the next section we discuss basic properties of the HDPSW with the help of a physically sound definition of the "mode effective area". Then, using waveguide parameters close to the optimum, we analyze several waveguide devices as directional couplers $[7,8,12]$, multimode interference couplers (MMI) [10], and the Mach-Zehnder interferometer based on the MMI couplers. For the full-vector 3D analysis of these structures we use our in-house modelling tools [13]-[16]. Results confirm that these structures represent promising building blocks of plasmonic devices, and also that our FMM codes can be efficiently used for full-vector 3D modelling of plasmonic devices.

\section{HYBRID DIELECTRIC-PLASMONIC SLOT WAVEGUIDES}

The cross-section of the hybrid dielectric-plasmonic slot waveguide being analyzed here is shown in Figure 1. The wave- 
(a)
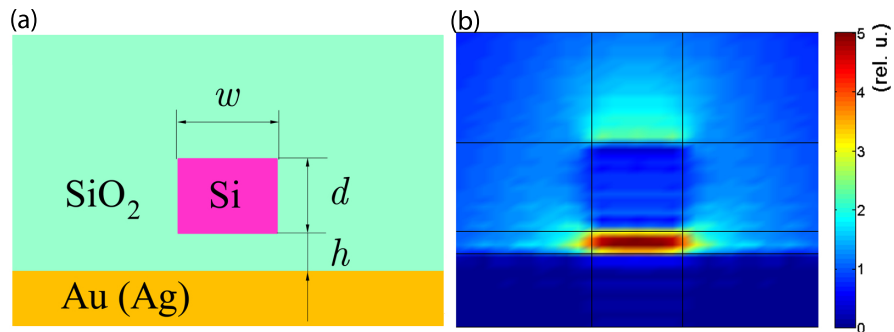

FIC. 1 a) Cross-section of a HDPSW, b) distribution of the absolute value of the vertical component of the electric field intensity of the fundamental mode for $w=150 \mathrm{~nm}$, $d=120 \mathrm{~nm}, h=30 \mathrm{~nm}$ at the wavelength of $1550 \mathrm{~nm}$.
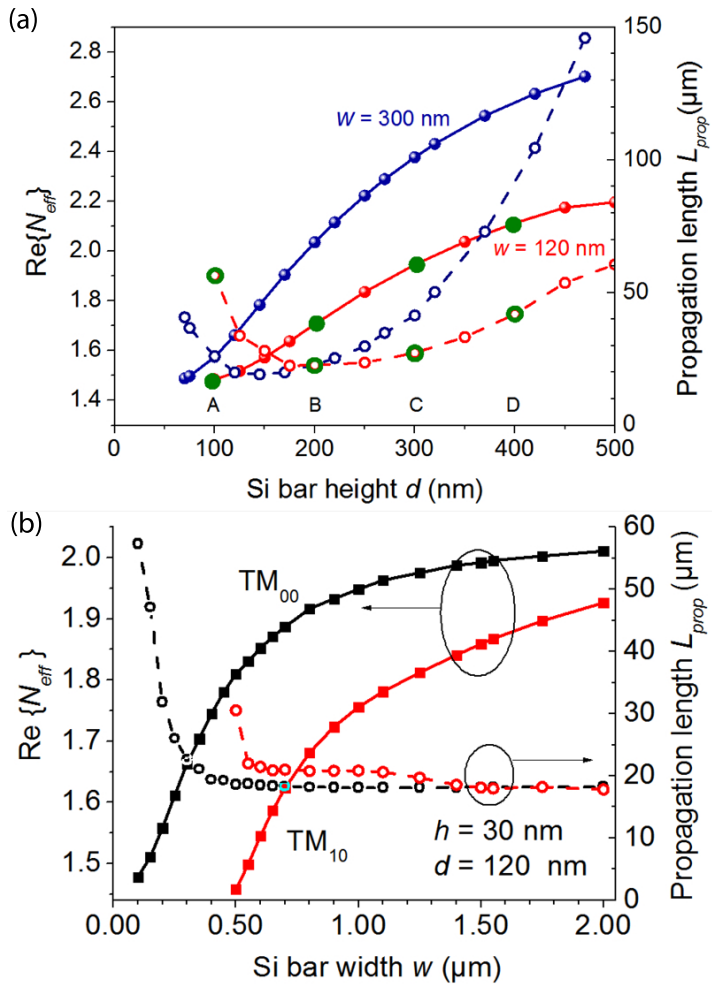

FIG. 2 The dependence of the real part of the effective refractive indices $\operatorname{Re}\left(N_{\text {eff }}\right)$ (solid lines) and the propagation lengths $L_{\text {prop }}$ (dashed) of the eigenmodes of the HDPSW on the waveguide parameters $d(\mathrm{a})$ and $w(\mathrm{~b})$. The gap size is $h=30 \mathrm{~nm}$.

guide consists of a high-index "photonic wire" separated from a metal surface by a very small low-index gap. In our design, the HDPSW is composed of a silicon nanowire surrounded by $\mathrm{SiO}_{2}$, and gold is considered as a metal layer. Optical properties of $\mathrm{Si}$ and $\mathrm{SiO}_{2}$ are well-known, and optical constants of e-beam vacuum-deposited Au layers were ellipsometrically measured in our laboratory. At the wavelength of $1550 \mathrm{~nm}$ we obtained $\varepsilon_{A u}=-117+6.6 i$. Substitution of $\mathrm{Au}$ with $\mathrm{Ag}$ makes only minor difference, however.

To get an insight into the properties of this waveguide, we plot the dependence of the real parts of the effective refractive indices $\Re \mathrm{e}\left\{N_{\text {eff }}\right\}$ and of the propagation lengths of the fundamental and higher-order TM modes on the silicon bar height $d$ and width $w$ in Figures 2(a),(b), respectively. The propagation length is defined as in [19],

$$
L_{\text {prop }}=\lambda /\left(4 \pi \cdot \Im \mathrm{m}\left\{N_{e f f}\right\}\right) .
$$

After a series of calculations of field distributions and propagation lengths for different values of the gap size $h$, we fixed
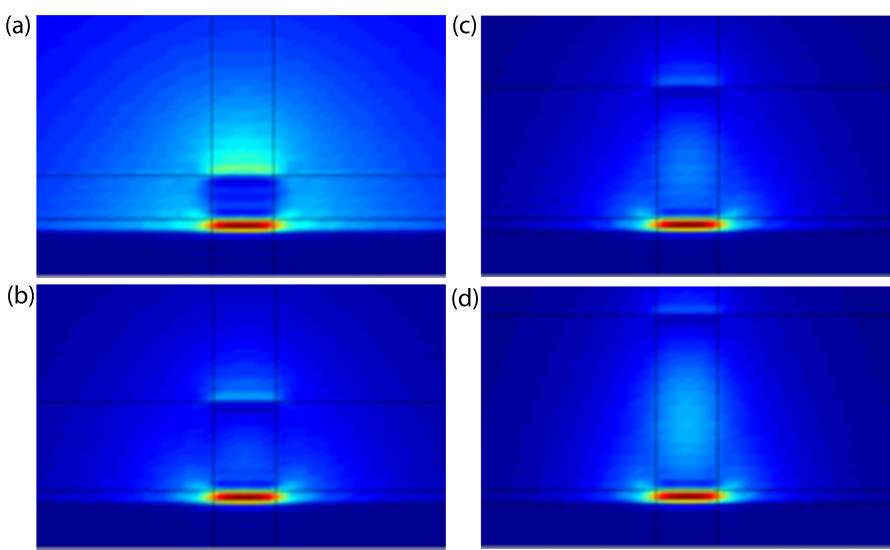

FIG. 3 Distributions of the absolute value of the vertical component of the electric field intensity of the fundamental eigenmode of HDPSW with $w=150 \mathrm{~nm}$ and $h=30 \mathrm{~nm}$ : a) $d=100 \mathrm{~nm}$, b) $d=200 \mathrm{~nm}$, c) $d=300 \mathrm{~nm}$, and d) $d=400 \mathrm{~nm}$. The colour scale is the same as in Figure $1(\mathrm{a})$.

the gap size at the value of $30 \mathrm{~nm}$. All calculations were performed for the wavelength $\lambda=1550 \mathrm{~nm}$. It is apparent that the real part of the effective refractive index is increasing with increasing both $d$ and $w$, and for $w \geq 500 \mathrm{~nm}$ the waveguide supports propagation of the higher lateral mode. The physical reason for the nonmonotonneous dependence of the propagation length on the silicon bar height $d$ in Figure 2(a) is apparent from Figure 3 that shows the distributions of the vertical component of the electric field intensity of the fundamental mode for four values of $d$ marked in Figure 2(a) with green dots, namely $d=100,200,300$ and $400 \mathrm{~nm}$.

It is apparent that for small $d$ below about $200 \mathrm{~nm}$, the field penetrates above the high-index bar, the field confinement is lower and the propagation length is correspondingly increased. For $d$ large enough, the field starts to concentrate in the high-index bar which again leads to reduced field confinement and increased propagation length. This behaviour can be reasonably quantified by using of the concept of the "effective mode area" for which we choose the "momentum definition"

$$
A_{e f f}=\frac{\pi \iint_{S} \Re \mathrm{e}\left(\mathbf{E} \times \mathbf{H}^{*} \cdot \mathbf{z}^{0}\right)\left(\mathbf{r}_{\perp}-\overline{\mathbf{r}}_{\perp}\right)^{2} d S}{\iint_{S} \Re \mathrm{e}\left(\mathbf{E} \times \mathbf{H}^{*} \cdot \mathbf{z}^{0}\right) d S},
$$

where

$$
\overline{\mathbf{r}}_{\perp}=\frac{\iint_{S} \Re \mathrm{e}\left(\mathbf{E} \times \mathbf{H}^{*} \cdot \mathbf{z}^{0}\right) \mathbf{r}_{\perp} d S}{\iint_{S} \Re \mathrm{e}\left(\mathbf{E} \times \mathbf{H}^{*} \cdot \mathbf{z}^{0}\right) d S}
$$

is the "center of gravity" of the power transmitted by the waveguide, $\mathbf{r}_{\perp}=(x, y)$ is the transverse position vector, $\mathbf{z}^{0}$ is a unit vector in the direction of propagation $z$, and $S$ is the total cross-section area of the waveguide. The dependence of the effective mode area on the high-index bar width plotted in Figure 4 agrees qualitatively well with the HDPSW behaviour demonstrated in Figures 2 and 3: for Si bar height $d=120 \mathrm{~nm}$ the effective mode area $A_{e f f}$ is small for widths $w$ between 200 and $300 \mathrm{~nm}$. 


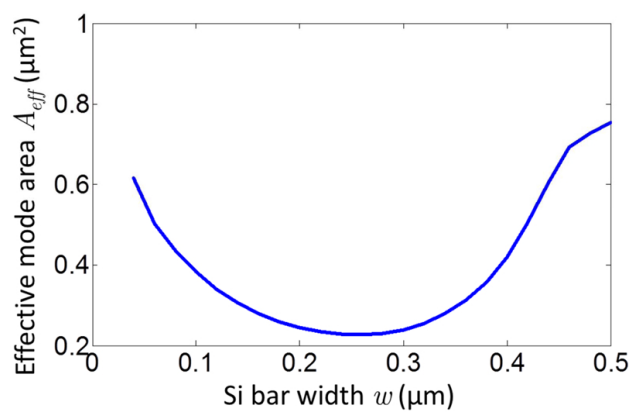

FIG. 4 Effective mode area of the HDPSW in dependence of the width $w$ of the highindex bar. $d=120 \mathrm{~nm}, h=30 \mathrm{~nm}, \lambda=1500 \mathrm{~nm}$.

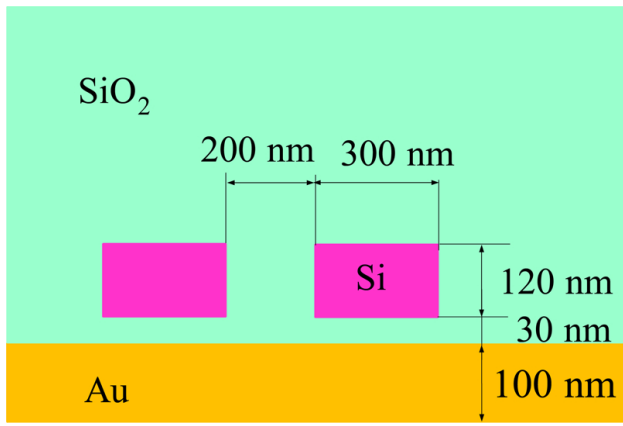

FIG. 5 The cross-section of the coupling section of the DC coupler.

\section{PLASMONIC WAVEGUIDE DEVICES}

In this section we briefly discuss three kinds of plasmonic devices based on HDPSW - a directional coupler (DC) [7, 8], a multimode interference (MMI) 3-dB coupler [10], and a MachZehnder interferometer based on 3-dB MMI splitters.

Let us start with directional couplers. Suitable waveguide dimensions in the coupling region of the DC can be chosen on the basis of Figure 1. To make use of a very strong field confinement in the HDPSW, we chose the slot width $h=30 \mathrm{~nm}$ and the high-index bar height $d=120 \mathrm{~nm}$. A reasonable choice of the waveguide structure in the coupling region should assure that individual waveguides are single mode while the twice wider waveguide supports propagation of just two modes. According to Figure 1(b), a suitable choice is apparently $w=300 \mathrm{~nm}$. In order to keep the coupling length reasonably short, we choose rather small gap between the waveguides, $g=200 \mathrm{~nm}$. The cross-section of the DC coupling section is schematically shown in Figure 5.

To allow low-crosstalk coupling to individual waveguides, input and output s-bends that increase the waveguide separation are required. We thus first analyzed the properties of an s-bend of the waveguide with $w=300 \mathrm{~nm}, h=30 \mathrm{~nm}$, and $d=120 \mathrm{~nm}$. Its shape was determined as a $5^{-t h}$-order polynomial which allows connecting any two given points in the waveguide plane with predetermined first and second derivatives at the initial points, i.e., the slopes and the curvatures of the bend. In our case, the slopes and curvatures were chosen to be zero, and in such case the bend shape is uniquely determined by its length and the lateral shift of the waveguide. We chose the s-bend length of $5 \mu \mathrm{m}$, and the lateral shift was chosen as $1 \mu \mathrm{m}$. The edge-to-edge separation of the
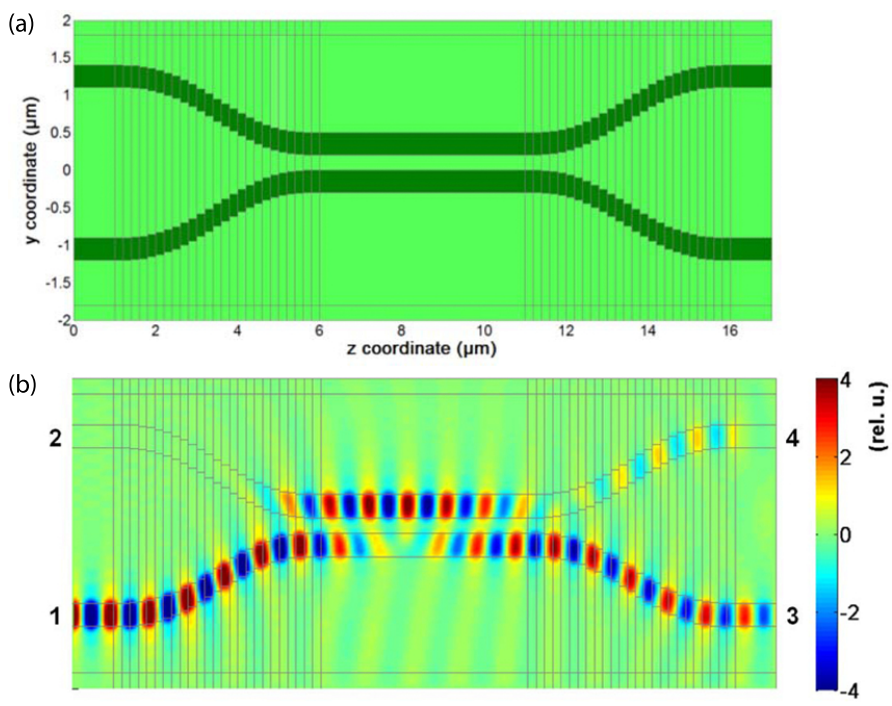

FIG. 6 a) The DC waveguide geometry, b) distribution of the absolute value of the vertical component of the electric field intensity within the plane $15 \mathrm{~nm}$ above the metal.

input and output waveguides is thus $2.2 \mu \mathrm{m}$ which safely prevents the waveguides from mutual coupling. Finally, we chose the length of the coupling section also as $5 \mu \mathrm{m}$. The waveguide geometry is plotted in Figure 6(a). As it is apparent, for the purpose of numerical modelling using our FMM algorithms that are based on eigenmode expansion and bidirectional propagation of eigenmodes in piecewise longitudinally uniform structures, the s-bends have to be approximated by staircase shapes. We found that reasonably reliable results can be obtained with the number of uniform segments in the input and output s-bend sections as low as 20 (shown in Figure 6(a).

In Figure 6(b) we demonstrate the field distribution in the DC structure excited into port 1 . Rather incidentally, at the wavelength of $1550 \mathrm{~nm}$, light is coupled just to the neighbouring waveguide and back. The power transmittance from port 1 to port 3 was calculated to be $20 \log \left|S_{31}\right|=-4.8 \mathrm{~dB}$. It can be estimated that to obtain a 3-dB splitting, the length of the coupling section should be as short as about $1.25 \mu \mathrm{m}$; the dominant device length is then primarily limited by input and output bends.

The feasibility of using also substantially longer devices is demonstrated in Figure 7 where we show the waveguide geometry and the field distribution in a DC with four times larger length of the coupling section $(20 \mu \mathrm{m})$. In this case, the power transmittance from port 1 to port 3 was calculated to be about $-9.4 \mathrm{~dB}$. As expected in plasmonic structures, the dominant loss is due to light absorption in a metal layer.

The next device that we describe here is a $1 \times 2$ splitter based on a multimode interference (MMI). We start with the same waveguide geometry of input and output ports, i.e., $w=300 \mathrm{~nm}, h=30 \mathrm{~nm}$, and $d=120 \mathrm{~nm}$. To keep the device small enough we chose the width of the MMI section only $2 \mu \mathrm{m}$. Then we modelled field distribution in the multimode section excited with the input waveguide and determined the suitable length of the MMI section and position of the output guides from this figure. The near-to-optimum length 

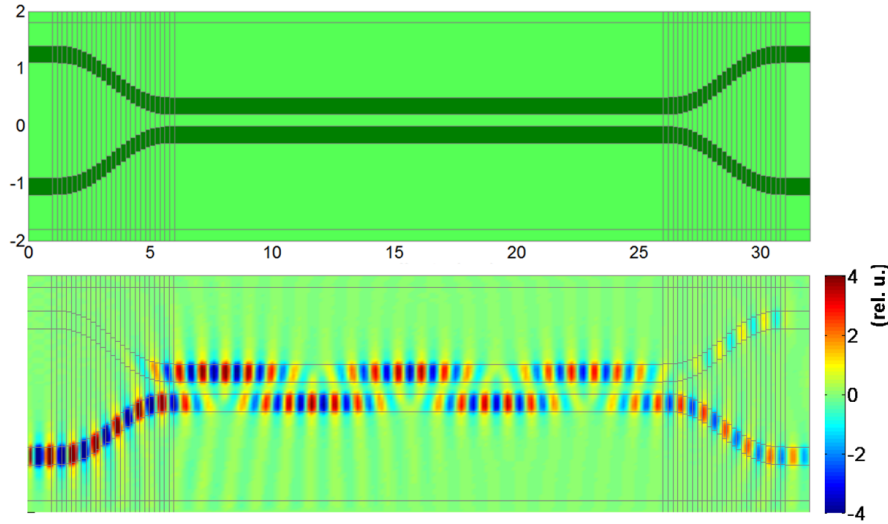

FIG. 7 The geometry and the vertical component of the electric field intensity distribution in the DC with the length of coupling section of $20 \mu \mathrm{m}$ ( $15 \mathrm{~nm}$ above the metal).

of the MMI section was thus determined as $3.5 \mu \mathrm{m}$, and the center-to-center separation of the output ports was $1.2 \mu \mathrm{m}$.

The MMI device design and the corresponding electric field distribution in the device are shown in Figure 8. Although the calculated power transmittance from port 1 to port 2 or 3 is reasonably high - about $-6 \mathrm{~dB}$, it is apparent from the standing wave pattern in the input waveguide that relatively high power is back-reflected from the MMI section; its calculated value is $-24 \mathrm{~dB}$. In order to suppress this back-reflection, we modified the structure by inserting short " $\lambda / 4$ impedance matching" sections between the ports and the MMI section. The sections were $500 \mathrm{~nm}$ wide and $250 \mathrm{~nm}$ long. The waveguide design and the calculated field distribution are shown in Figure 9. It is apparent that the standing-wave pattern in the input waveguide is now substantially weaker - the calculated reflectance was only $-51 \mathrm{~dB}$. The forward 1 to 2 (or 3) port transmittance was only weakly increased to $-5.5 \mathrm{~dB}$. In both Figures 8 and 9, it is also seen that inside the MMI section there are rather strong Fabry-Perot (F-P) interferences. We have not so far investigated this effect deep enough; we suppose that their origin comes from imperfect group velocity matching at the interfaces between the port waveguides and the MMI section.

Inspired with [10], we have also tried to reduce the dimensions of the MMI splitter. We found that reduction to about $1 / 2$ in both horizontal $(y, z)$ dimensions (i.e., to $1 / 4$ in the device area) is, in principle, possible, but in this case, the separation of the two output ports is too small: they are mutually coupled with the coupling length of about $5 \mu \mathrm{m}$, which drastically affects the application of the MMI coupler.

Rather promising operation of HDPSW MMI splitters led us to the idea to investigate the behaviour of a compact plasmonic Mach-Zehnder (MZ) interferometer based on these MMI splitters. Its waveguide structure is shown in Figure 10(a). In principle, it is formed by two MMI couplers connected together by the two arms. The length of both arms was chosen equal, of $20 \mu \mathrm{m}$. The field distribution in this symmetric MZ is shown in Fig. 10b). The calculated transmittance was $-6 \mathrm{~dB}$, the back-reflected power was at the level of $-37 \mathrm{~dB}$.

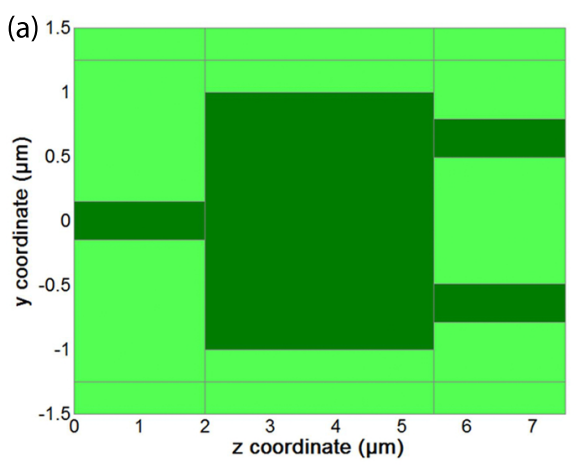

(b)

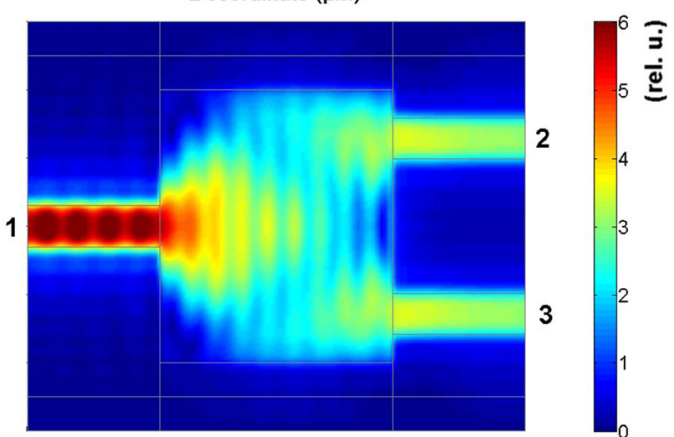

FIC. 8 First design of a MMI 3-dB splitter based on HDPSW. a) waveguide configuration, b) distribution of the absolute value of the vertical component of the electric field intensity.

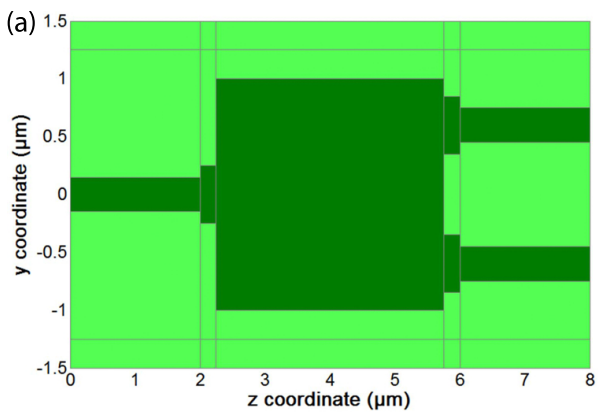

(b)

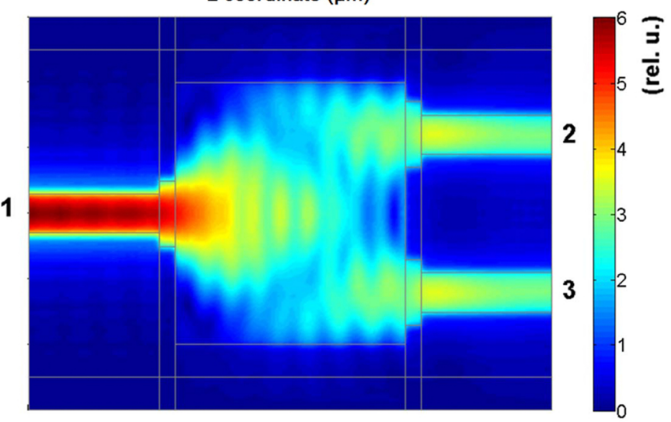

FIG. 9 Improved design of a MMI 3-dB splitter based on HDPSW. a) Waveguide configuration, b) distribution of the absolute value of the vertical component of the electric field intensity.

To demonstrate the behaviour of the unbalanced $\mathrm{MZ}$, we modified the refractive index within the slot between the highindex bar and the metal surface just below the arms in the opposite sense so as to reach the relative phase shift between the arms close to $\pi$. The required refractive index change was found to be rather huge, about \pm 0.15 . The calculated field distribution in this unbalanced $\mathrm{MZ}$ interferometer is plotted in Figure 10(c). It is apparent that throughput power is now "switched-off"; the calculated transmittance decreased to $-21 \mathrm{~dB}$. Higher extinction could be perhaps attainable by more careful tuning of the refractive index change, but it would 


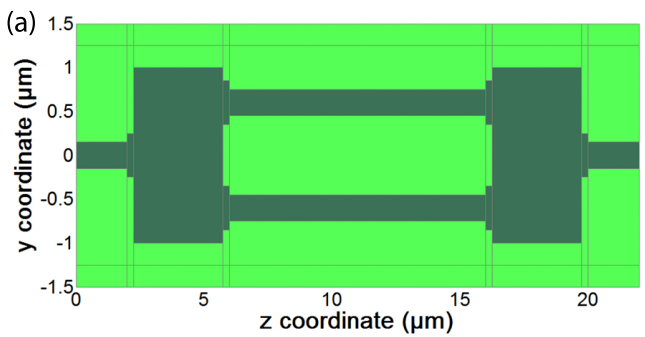

(b)

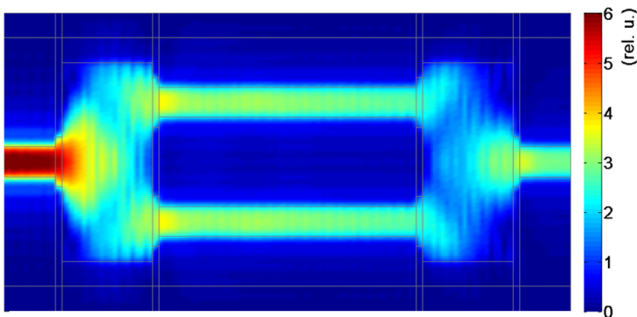

(c)

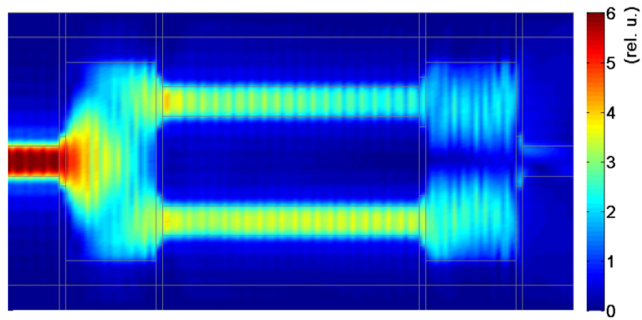

FIG. 10 HDPSW-based Mach-Zehnder interferometer. a) Waveguide structure, b) field distribution in the balanced $M Z, c$ ) field distribution in the unbalanced $M Z$.

be limited by the difference in attenuation of the arms due to their different refractive indices. Note that in this case, the reflected power increased to $-25 \mathrm{~dB}$, and F-P resonances are clearly visible also in the arms.

We have to concede that we have also tried to model HDPSW microring resonators similar to those described in [11], but without clear success - the calculated losses were too high and thus prohibitive for proper functioning of the device. The reason for this has to be analyzed in more detail which we plan to report in a succeeding paper.

\section{CONCLUSION}

In our contribution we analyzed a novel type of a plasmonic waveguide - the hybrid dielectric-plasmonic slot waveguide that combines a very high field confinement into a deeply subwavelength area with moderate loss. Propagation lengths are in the range of $50 \mu \mathrm{m}$ at the telecommunication wavelength of $1.55 \mu \mathrm{m}$. We have also numerically investigated several types of devices based on this type of waveguides - directional couplers, $3 \mathrm{~dB}$ MMI splitters, and a Mach-Zehnder interferometer using our 3-D codes developed in-house on the basis of the Fourier Modal Method. Although we have not tried to fully optimize the design of the devices, their basic functionality was clearly demonstrated. We believe that because of its compatibility with the present silicon microelectronics, this type of planar hybrid semiconductor-based plasmonic waveguide can find interesting applications, e.g., in on-chip data communication, optical sensing, etc.

\section{ACKNOWLEDGEMENTS}

This work has been financially supported by the Czech Science Foundation under Project P205/10/0046 and by the Ministry of Education, Youth and Sports of the Czech Republic under Projects OC09061 and OC09038.

\section{References}

[1] M. Z. Alam, J. Meier, J. S. Aitchison, and M. Mojahedi, "Super Mode Propagation in Low Index Medium," in Proceedings to CLEO/QELS 2007, JThD112 (CLEO, Baltimore, 2007).

[2] R. F. Oulton, V. J. Sorger, D. A. Genov, D. F. P. Pile, and $X$. Zhang, "A hybrid plasmonic waveguide for subwavelength confinement and long-range propagation," Nat. Photonics 2, 496-500 (2008).

[3] D. Dai, and S. He, "A silicon-based hybrid plasmonic waveguide with a metal cap for a nano-scale light confinement," opt. Express 17, 16646-16653 (2009).

[4] M. Fujii, J. Leuthold, and W. Freude, "Dispersion Relation and Loss of Subwavelength Confined Mode of Metal-Dielectric-Gap Optical Waveguides," IEEE Photonic. Tech. L. 21, 362-364 (2009).

[5] Y. Binfeng, H. Guohua, J. Yang, and C. Yiping, “Characteristics analysis of a hybrid surface plasmonic waveguide with nanometric confinement and high optical intensity," J. Opt. Soc. Am. B 26, 1924-1929 (2009).

[6] R. F. Oulton, V. J. Sorger, T. Zentgraf, R.-M. Ma, C. Gladden, L. Dai, et al., "Plasmon lasers at deep subwavelength scale," Nature 461, 629-632 (2009).

[7] H.-S. Chu, E.-P. Li, P. Bai, and R. Hedge, "Optical performance of single-mode hybrid dielectric-loaded plasmonic waveguide-based components," Appl. Phys. Lett. 96, 221103-1-3 (2010).

[8] J. Tian, Z. Ma, Q. Li, Y. Song, Z. Liu, Q. Yang, et al., "Nanowaveguides and couplers based on hybrid plasmonic modes," Appl. Phys. Lett. 97, 231121-1 (2010).

[9] H.-S. Chu, Y. A. Akimov, P. Bai, and E.-P. Li, “Hybrid dielectricloaded plasmonic waveguide and wavelength selective components for efficiently controlling light at subwavelength scale," J. Opt. Soc. Am. B 28, 2895-2901 (2011).

[10] J. Wang, X. Guan, Y. He, Y. Shi, Z. Wang, S. He, et al., "Sub- $\mu \mathrm{m}^{2}$ power splitters by using silicon hybrid plasmonic waveguides," Opt. Express 19, 838-847 (2011).

[11] H.-S. Chu, Y. Akimov, P. Bai, and E.-P. Li, "Submicrometer radius and highly confined plasmonic ring resonator filters based on hybrid metal-oxide-semiconductor waveguide," Opt. Lett. 37, 4564-4566 (2012).

[12] F. Lou, Z. Wang, D. Dai, L. Thylen, and L. Wosinski, “Experimental demonstration of ultra-compact directional couplers based on silicon hybrid plasmonic waveguides," Appl. Phys. Lett. 100, 241105-1-4 (2012).

[13] J. Čtyroký, "Improved bidirectional-mode expansion propagation algorithm based on fourier series," J. Lightwave Technol. 25, 2321-2330 (2007).

[14] J. Čtyroký, P. Kwiecien, and I. Richter, "Fourier Series-Based Bidirectional Propagation Algorithm with Adaptive Spatial Resolution," J. Lightwave Technol. 28, 2969-2976 (2010).

[15] J. Čtyroký, "3-D Bidirectional Propagation Algorithm Based on Fourier Series," J. Lightwave Technol. 30, 3699-3708 (2012). 
[16] J. Čtyroký, P. Kwiecien, and I. Richter, "Fourier Series Based 3D Bi-directional Propagation Algorithm for Integrated Photonics," in Proceedings of the $18^{\text {th }}$ European Conference on Integrated Optics ECIO 2012 (CIRSE, Barcelona, 2012).

[17] J. Jágerská, N. Le Thomas, R. Houdré, J. Bolten, C. Moormann, T. Wahlbrink, et al., "Dispersion properties of silicon nanophotonic waveguides investigated with Fourier optics," Opt. Lett. 32, 2723-2725 (2007).
[18] H. Benisty, and M. Besbes, "Plasmonic inverse rib waveguiding for tight confinement and smooth interface definition," J. Appl. Phys. 108, 063108-1-8 (2010).

[19] P. Berini, "Figures of merit for surface plasmon waveguides," Opt. Express 14, 13030-13042 (2006). 